\title{
Histological analysis of anti-cancer drug loaded, targeted Mn:ZnS quantum dots in metastatic lesions of $4 \mathrm{~T} 1$ challenged mice
}

\begin{abstract}
5-Fluororaucil (5-FU) as anti-cancer drug was reported to induce thymidine synthase (TS) overexpression and cancer cell resistance. To improve its therapeutic efficacy and selective targeting, here we developed a targeted delivery system mediated by the active ligand-folate receptor chemistry to deliver the 5-FU drug selectively into the tumor microenvironment. The preparation was achieved by exploring chitosan (CS)-biopolymer based system with folic acid (FA)-conjugation. The 5-FU@FACS-Mn:ZnS quantum dots (QDs) based on the histological assessment conducted in the 4T1 challenged mice showed an improved tumor remission in the liver, spleen and lungs. The 5-FU@FACS-Mn:ZnS composite induced antiproliferative properties in these organs as compared to the free 5-FU drug. Unlike the 5FU@FACS-Mn:ZnS treated groups which showed some specific morphological changes such as cell shrinkage without obvious presence of adipocytes, the excised section of the tumor in the untreated control group and the free 5-FU drug treated group showed necrotic and degenerated cells; these cells are multifocally distributed in the tumor mass with evidence of widely distributed adipocytes within the tumor mass. These findings suggest that the 5-FU@FACS-Mn:ZnS composite has a superior role during the induction of apoptosis in the 4T1 cells as compared to the free 5-FU drug treated groups. The results of the study therefore suggest that the impregnation of 5-FU anti-cancer drug within the FACS-Mn:ZnS system significantly improves its selective targeting efficacy, in addition to improving the anti-proliferative properties and attenuate possible tumor resistances to the 5-FU drug.
\end{abstract}

Keyword: Histological analysis; Anti-cancer drug; Cancer cell resistance; Mice 